\title{
Purcell factor enhanced scattering efficiency in silicon nanocrystal doped micro-cavities
}

\author{
T. J. Kippenberg ${ }^{1}$, A. Tschebotareva ${ }^{2}$, J. Kalkman², A. Polman ${ }^{2}$, K.J. Vahala ${ }^{1}$ \\ 1) Department of Applied Physics, California Institute of Technolog \\ 2) F.O.M. Institute, AMOLF, Netherlands
}

\begin{abstract}
:
Scattering induced by nano-particles in a microcavity is investigated for the case of silicon nanocrystal doped microtoroids and a significant enhancement of scattering into the originally doubly-degenerate cavity eigenmodes is found, exceeding $>99.42 \%$.

(C)2000 Optical Society of America

OCIS codes:, (230.5750) Resonators
\end{abstract}

It is a well known phenomenon ${ }^{1,2}$ that the resonances of whispering gallery mode (WGM) cavities such as droplets, microspheres, microdisks and microtoroids appear as doublets. The doublet splitting is due to lifting of the two-fold degeneracy of the clockwise $(\mathrm{CW})$ and counter-clockwise $(\mathrm{CCW})$ whispering gallery modes, which can be induced by scattering, either by intrinsic or surface defect centers. Yet, the efficiency of this mutual coupling process, has, until now, not been investigated. Here, we present for the first time a measurement of the capture efficiency of this process, for the case of silicon nanocrystal doped microcavities. A novel measurement technique is introduced which allows to infer the exact capture efficiency in the presence of other loss channels and enables differentiation between losses due to absorption and scattering. Using this method we show that dramatic enhancement of scattering efficiency is possible in a high-Q small mode volume (or equivalently high-Purcell factor) micro-cavity, and experimentally demonstrate a capture efficiency of $99.42 \%$. This observation implies that preferential scattering of light into the original cavity eigenmodes occurs leading to a reduced scattering loss as measured by the $\mathrm{Q}$ factor. The observed findings are explained by considering the role of the optical density of states (DOS) in the scattering process, and a classical analysis shows that the scattering enhancement is related to the enhancement of DOS over the free space value which is recognized as the well-known Purcell factor. Our results show that the enhanced density of states not only affects the lifetime and the spatial emission profile of an excited atom inside a cavity (i.e. a quantum dipole), but also has a profound affect on the spatial emission pattern of a classical dipole (e.g. a scattering center). As such, our results establish for the first time to the author's knowledge a connection between the Purcell factor and scattering of a nanoparticle's within a microcavity.

The presence of nano-particles in a microcavity mode gives rise to scattering, whose rate is given by $\sigma_{t o t}=\sigma_{\text {scat }} N$,

where $\mathrm{N}$ is the density of nanoparticles and $\sigma_{\text {scat }}$ the individual nano-particle scattering cross section. Part of the scattered light will be scattered into non-cavity eigenmodes and increase dissipation, thereby decreasing the $Q$ factor. In contrast, the part of the light scattered into the original pair of $\mathrm{CW}$ and $\mathrm{CCW}$ eigenmodes, does not cause excess dissipation, but induces modal coupling (i.e. the splitting of a single resonance into a doublet). If we designate $\eta$ to be the capture efficiency into both the $\mathrm{CW}$ and $\mathrm{CCW}$ mode, the presence of nano-particles causes an observable mode splitting of:

$$
\gamma^{-1}=\frac{1}{2} \eta \frac{c}{n} \sigma_{\text {scat }} N
$$

The remaining part of the scattered light causes excess cavity dissipation as given by

$$
\tau^{-1}=(1-\eta) \frac{c}{n} \sigma_{\text {scat }} N
$$

The factor of $1 / 2$ in the equation (1) takes into account that the scattering of light into original eigenmodes (i.e., selfcoupling) does not contribute to the observed mode splitting (and light is scattered equally into CW and CCW direction). The degree to which the scattering process couples WGM-system can be described by the modal coupling parameter $(\Gamma)^{3}$, which effectively gives the doublet visibility (as defined by the ratio of splitting frequency to intrinsic linewidth). Significantly, this parameter is also experimentally easily accessible, by fitting the observed doublet structure to a coupled oscillator model as described in reference ${ }^{1,3}$. In what follows we analyze how the capture efficiency $(\eta)$ can be retrieved from the visibility of the doublet structure. In the presence of other loss channels, such as residual material absorption (expressed by $1 / \tau$ ), the $\Gamma$-parameter can be expressed as: 


\section{QME6}

$$
\Gamma=\left(\frac{\frac{1}{2} \eta \gamma_{t o t}^{-1}}{\tau_{0}^{-1}+(1-\eta) \gamma_{t o t}^{-1}}\right)
$$

This equation relates the efficiency of the scattering process to the observed doublet visibility. It is also clear that if the residual absorption is known and is constant (i.e. an intrinsic property of the resonator), the actual scattering efficiency $(\eta)$ can be inferred exactly. Notably, if the modal coupling parameter $(\Gamma)$ is known for various scattering contributions $(\gamma)$, the functional dependence of the Q- $\Gamma$ data can be used to accurately infer the efficiency $\eta$, via the functional relation of $\Gamma(\mathrm{Q}, \gamma)$ given by equation (1).

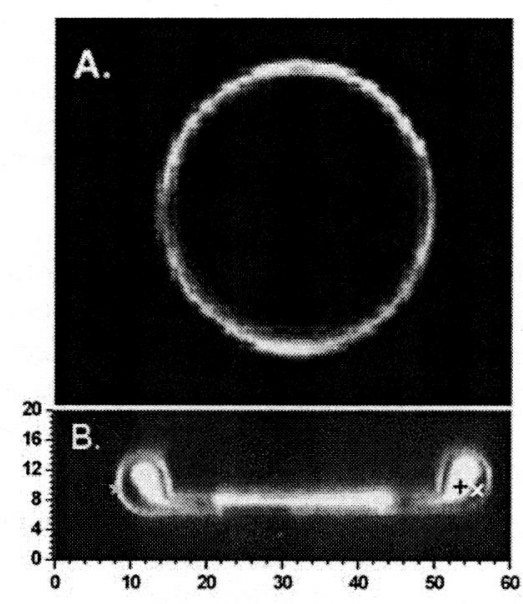

Figure 1: Confocal luminescence imaging of a 50-micron-diameter silicon nanocrystal doped toroid microcavity (inner diameter ca. $\sim 7$ micron). Upper image: 2-D image obtained by scanning the plane containing the toroid. The luminescence (color-coded) is recorded in the $650-750 \mathrm{~nm}$ band. The peak emission wavelength within the toroid was $\sim 680 \mathrm{~nm}$, which corresponds to $3.5 \mathrm{~nm}$ silicon nanocrystals. Lower graph: Cross sectional scan through the toroid, in the presence of an rhodamine doped index matching gel. The image is recorded in the spectral domain of $650-750 \mathrm{~nm}$, and clearly reveals the presence of nanocrystals in the toroid.

To test the above model, we have investigated the scattering processes of toroid microcavities embedded with silicon nano-crystals on a chip. Microtoroids exhibit ultra-high-Q whispering-gallery type modes [9], and can be used as efficient nonlinear Raman lasers, optical parametric oscillators, ultra-low loss filters or erbium microlasers. Details of the fabrication of these structures and on the coupling mechanism, which employs tapered optical fibers, can be found in references ${ }^{4-6}$. We proceeded to increase the scattering contribution by using silicon nanocrystal doped microcavities. Silicon nanocrystals (Si nc's) are nanometer sized crystalline silicon aggregates exhibiting quantum-confined photoluminescence in the visible and having potential applications in electronics and photonics. ${ }^{7}$ Since Si-ncs do not absorb at 1.5 micron and have a high index contrast to the silica matrix, they act as strong scatterers inside the microcavity and induce a transition into the scattering-limited regime.

Silicon nanocrystals were obtained by implanting $900 \mathrm{keV} \mathrm{Si}+$ ions into a $2 \mu \mathrm{m}$ thick layer of $\mathrm{SiO} 2$ thermally grown on a $\mathrm{Si}$ wafer, and performing a subsequent anneal of the sample at $1100^{\circ} \mathrm{C}$ for $10 \mathrm{~min}$ in Ar gas flow. To confirm the presence of nanocyrstals in the silica microcavity, we performed 3-D confocal photoluminescence (PL) studies. For this purpose, the microtoroid was covered by a layer of rhodamine and embedded into standard immersion oil with refractive index of 1.52. Figures 1(a) and 1(b) show the integrated intensity of the nanocrystal emission of the toroidal microcavity in the XY-plane and in the plane YZ-plane. These figures clearly demonstrate the presence of nanocrystals and in addition that the Si nanocrystals are located in the toroidal ring. 

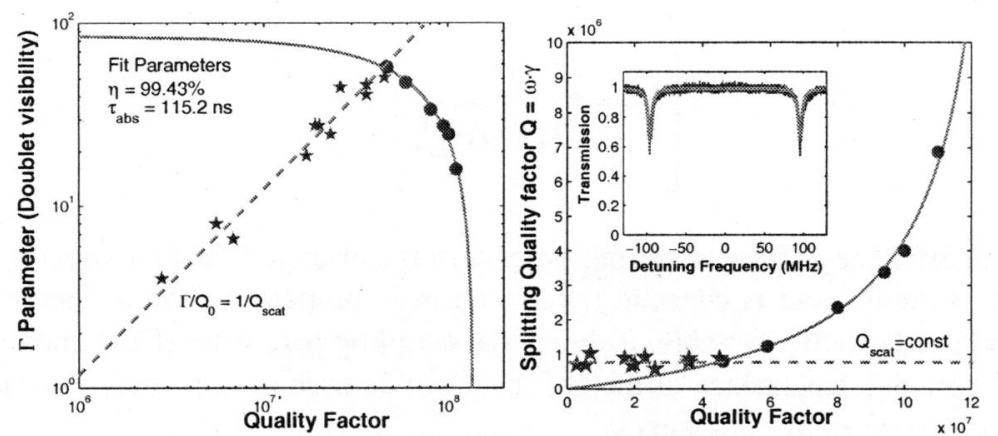

Figure 2. Right graph: Quality factor versus Gamma parameter for a silicon nanocrystal doped $\mathrm{SiO} 2$ microcavity (geometry: inner diameter $\sim 7$ micron, outer diameter $\sim 50$ micron). The solid line in both graphs is a fit using equation (1) to the scattering-limited WGMs (circles), under the assumption of constant residual background absorption. A two parameter fit yields an absorption lifetime of $\tau$ abs $=115$ ns lifetime, and a backscattering efficiency of $\eta=99.42 \%$. The dashed curve and corresponding data (and flat line with corresponding data in upper graph) are absorption limited points provided by higher order radial modes. Right graph: Splitting Quality factor (i.e. versus scattering-Q for the same sample. The inset shows a typical mode splitting in the Si:nc doped sample, exhibiting a modal coupling parameter of 40 . Solid line is a fit of the scattering limited WGMs using equation 3.

To measure the scattering properties of silicon nanocrystals, we measured the doublet visibility (as given by the $\Gamma$ parameter) in the under-coupled regime, by fitting the doublet to a coupled harmonic oscillator model, as is described in detail in ref. ${ }^{3}$. In order to obtain an exact value of the scattering efficiency, the first three azimuthal modes were excited and measured for progressing angular mode numbers (1). These modes are dominated by (differing levels) of scattering (by the randomly nucleated nanocrystals), but experience the same amount of residual absorption (due to the presence of $\mathrm{OH}$ adsorption on the surface of the toroid). The results of these measurements are shown in Figures 2(a) and 2(b). The solid line is a two-parameter fit ( $\eta$ and $\tau 0$ ) of equation (1) applied to the experimental data, and excellent agreement is obtained. The lower Q data is believed to be caused by higher order radial modes, which possess increased WGM loss (and follow absorption-limited Q behavior). The intrinsic cavity lifetime retrieved from the fit to the data was $115 \mathrm{~ns}$. The fit also yields a remarkably high scattering efficiency of $99.42 \%$, which clearly demonstrates that scattering processes lead to preferential scattering into the original eigenmodes.

This remarkably high efficiency is explained by considered the role of DOS, in the scattering process, and an enhancement is found to be related to the enhancement of the microcavity DOS of the free space value -which is recognized as the Purcell factor. In the case of a microcavity we thus can assume that the scattering capture efficiency $\eta$ is given by $\eta=\frac{F}{F+1}$, where $\mathrm{F}$ is the Purcell-factor. For the present cavity this expression leads to an expected scattering efficiency in excess of $99.98 \%$ (corresponding to a Purcell factor of $\mathrm{F}=6300$ for $\mathrm{Q}=10^{8}$ and a mode volume of $\sim 1200 \lambda^{3} / \mathrm{n}^{3}$ ). However, the experimental values are much lower than the theoretical prediction (the corresponding Purcell factor being $\mathrm{F}=172$ ). To address this apparent discrepancy $(\mathrm{a}$ factor $\times 40)$ with our theoretical prediction, we note that in the above analysis we neglected the absorption cross section of the silicon nano-particles themselves. The result demonstrate that the dissipation in a cavity can be significantly lower than for a bulk material ${ }^{1}$, and establishes for the first time a connection between the Purcell-factor and scattering processes within a microcavity.

1. Gorodetsky, M. L., Pryamikov, A. D. \& Ilchenko, V. S. Rayleigh scattering in high-Q microspheres. Journal of the Optical Society of America B-Optical Physics 17, 1051-1057 (2000).

2. Weiss, D. S., Sandoghdar, V., Hare, J., Lefevreseguin, V., Raimond, J. M. \& Haroche, S. Splitting of High-Q Mie Modes Induced by Light Backscattering in Silica Microspheres. Optics Letters 20, 1835-1837 (1995).

3. Kippenberg, T. J., Spillane, S. M. \& Vahala, K. J. Modal coupling in traveling-wave resonators. Optics Letters 27, 1669-1671 (2002).

4. Armani, D. K., Kippenberg, T. J., Spillane, S. M. \& Vahala, K. J. Ultra-high-Q toroid microcavity on a chip. Nature 421, 925-928 (2003).

5. Cai, M., Painter, O. \& Vahala, K. J. Observation of critical coupling in a fiber taper to a silica- microsphere whispering-gallery mode system. Physical Review Letters 85, 74-77 (2000).

6. Spillane, S. M., Kippenberg, T. J., Painter, O. J. \& Vahala, K. J. Ideality in a fiber-taper-coupled microresonator system for application to cavity quantum electrodynamics. Physical Review Letters 91, art. no.-043902 (2003).

7. Polman, A. Photonic materials - Teaching silicon new tricks. Nature Materials 1, 10-12 (2002). 